\title{
A New Method to Improve Round Robin Scheduling Algorithm with Quantum Time Based on Harmonic-Arithmetic Mean (HARM)
}

\author{
Ashkan Emami Ale Agha \\ Department of Computer Engineering, Science and Research Branch, Islamic Azad University, Tehran, Iran \\ E-mail: ashkan.eaa@Gmail.com
}

\author{
Somayyeh Jafarali Jassbi \\ Assistant Professor, Department of Computer Engineering, Science and Research Branch, Islamic Azad University, \\ Tehran, Iran \\ E-mail: s.jassbi@sr.iau.ac.ir
}

\begin{abstract}
One of the most important concepts in multi programming Operating Systems is scheduling. It helps in choosing the processes for execution. Round robin method is one of the most important algorithms in scheduling. It is the most popular algorith $m$ due to its fairness and starvation free nature towards the process es, which is achieved by using proper quantum time. The main challenge in this algorithm is selection of quantum time. This parameter affects on average Waiting Time and average Turnaround Time in execution queue. As the quantum time is static, it causes less context switching in case of high quantum time and high context switching in case of less quantum time. Increasing context switch leads to high average waiting time, high average turnaround time which is an overhead and degrades the system performance. With respect to these points, the algorith ms should calculate proper value for the quantum time. Two main classes of algorithms that are proposed to calculate the quantum time include static and dynamic methods. In static methods quantum time is fixed during the scheduling. Dynamic algorithms are one of these methods that change the value of quantum time in each cycle. For example in one method the value of quantum time in each cycle is equal to the median of burst times of processes in ready queue and for another method this value is equal to arithmetic mean of burst times of ready processes.
\end{abstract}

In this paper we proposed a new method to obtaining quantum time in each cycle based on arithmeticharmonic mean (HARM). Harmonic mean is calculated by dividing the number of observations by the reciprocal of each number in the series. With examples we show that in some cases it can provides better scheduling criteria and improves the average Turnaround Time and average Waiting Time.
Index Terms - Round Robin, Quantum Time, Harmonic Mean, Arithmetic Mean, HARM, Average Waiting Time, Average Turnaround Time

\section{Introduction}

Operating system is the interface between the hardware and the application programs. It simplifies the use of the hardware and hides its complexity from user. Modern operating systems have become more complex, they have evolved from a single task to a multitasking environment in which processes run in a concurrent manner [11, 12]. In multitasking and multiprocessing environment the way the processes are assigned to run on the available CPUs is called scheduling. In multi programming systems which several applications are executed simultaneously there should be a fairly scheduling mechanism to share the CPU between processes [13]. Scheduling is the method by which threads; processes are given access to processor. In Real-Time multiprogramming systems there are different methods to schedule processes [14]. There are several scheduling algorithms in operating systems. Each scheduling algorith $m$ has its own advantages and disadvantages. Round Robin is one of the most widely used CPU scheduling algorithms. The Round Robin scheduling algorithm is designed especially for time sharing systems. It is similar to First Come First Serve (FCFS) scheduling, but preemption is added to switch between processes. A small unit of time, called a quantum time or slice time, is defined. The main problem in Round Robin algorithm is selection of appropriate quantum time. If the quantum time is very small, it increases the number of context switches and if the quantum time is very large it leads to increasing of waiting time. The proposed method in [3] calculates quantum time in each cycle based on average of remain ing burst times. In cases where the burst time of new process has large difference from previous processes, depending on how much smaller or how 
much larger than the previous ones, arith metic mean or harmonic mean can be used. In this paper we show that in what situations it is better to use arith metic mean and in what situation it is better to use harmonic mean. Harmonic mean is quotient of number of the given values and sum of the reciprocals of the given values [16]. All burst times are greater than zero, so we can use harmonic mean to calculate the quantum times. We have experimentally shown that our proposed method is better than previous methods by reducing average Waiting Time and average Turnaround Time.

\section{Previous Works}

Round Robin is used in almost every operating system and for this reason many algorithms are proposed by researchers to enhance this algorithm. The static quantum time which is a limitation of Round Robin was removed by taking dynamic quantum time.

Matarneh [1] suggested that to optimize the quantum time, it can be calculated by median of burst times for the set of processes in ready queue. In [2] the optimized quantum time is selected by calculating the mean value burst time of sorted processes. The proposed method in [3] calculates quantum time in each cycle based on average of remaining burst times. Helmy and Dekdouk in [4] have employed a weighting method to combine low scheduling overhead of round robin algorithms and favor short job. Rakesh Mohanty and Behera in [5] have proposed SRBRR method that calculates smart quantum time by the median of the increasingly sorted burst time of all the processes. Fittest Job first Dynamic Round Robin (FJFDRR) proposed by Rakesh Mohanty and Manas [6] calculates quantum time in each cycle by considering three parameters: user priority, burst time and arrival time. A new fit factor is calculated in each cycle which decides the sequence of the execution of the processes. The algorithm that has been suggested by Saroj Hiranwal in [7] based on smart time slicing which depends on three aspects they are priority, average CPU burst or mid process CPU burst, and context switch avoidance time.Pallab and probal in [10] suggested that quantum time is the mean of the summation of the average and the maximum burst time. In other works, smart quantum time is used to calculate quantum time.

\section{Organization of the Paper}

In section 4, researcher's motivation to propose this algorith $\mathrm{m}$ has been considered. Illustration and pseudo code of our algorith m (HARM) is presented in section 5 . In section 6, experimental results of our new algorithm by studying data from several tables and comparing them with previous algorithms are shown. Conclusion and directions for future works is given in section 7 .

\section{Motivation}

In many fields that Round Robin algorithm can be used, quantum times of processes are close together because of the nature of those fields. For example, consider a cell phone with many background processes such as CrashMonitor and DeviceMonitor and clock. Such processes are always active except when the device is turned off or the user disable them. Thus the burst times of these processes are close together and approximately is equal to the time between two consecutive boots of device. But in some applications the burst times of processes are not close together. For example in a network with one server there are many users with completely different tasks from editing a large file to execute a simple command in Command Prompt of server. Our studies showed that for this type of processes the use of round robin algorithm based on arithmetic mean does not provide optimal results. For this reason we decided to design an improved algorithm to resolve above problems. Our new suggested algorithm provides better average Waiting Time and average Turnaround Time.

\section{Proposed Method}

In this paper quantum time in each cycle is calculated based on Harmonic-Arith metic mean (HA RM) which is useful when burst times of processes are heterogeneous and some of them have very difference from the others. Harmonic mean is another measure of central tendency and also based on mathematic footing like arithmetic mean and geometric mean. Shannon Pratt has understated the propriety for the use of the harmonic mean value of an array of ratios as opposed to the arith metic mean value or median as "a very attractive alternative measure of central tendency". According to Shannon Pratt, "Although the harmonic mean is not used frequently, probably because it is unfamiliar to most readers of valuation reports, it is conceptually a very attractive alternative measure of central tendency"[8,9]. Harmonic mean has some interesting properties. First; it can be calculated for numbers greater than zero and non zero. Second, tends toward very small quantities. Harmonic mean is one of the three pythagoreans averages. For all sets that contain at least one pair of unequal value, the harmonic mean is the lowest value of them, whereas the arith metic mean is always highest of them and the geometric mean is always between them. It makes a most dramatic appearance in the problem of average speed, but also pops up in various geometric circumstances.

\subsection{Harmonic-Arithmetic Mean (HARM) Round Robin Algorithm}

Suppose that we have $n$ processes with burst time $t_{1}$, $t_{2}, t_{n}$. Harmonic mean of these values is shown in 
equation 1 and arith metic mean of these values is shown in equation 2[15].

$$
\begin{aligned}
& \text { HarmonicMean }=\frac{n}{\frac{1}{t_{1}}+\frac{1}{t_{2}}+\ldots+\frac{1}{t_{n}}} \\
& \text { ArithmeticMean }=\frac{t_{1}+t_{2}+\ldots+t_{n}}{n}
\end{aligned}
$$

Harmonic mean has many applications in computer science such as machine learning and information retrieval $[16,17]$.

Here we discuss about harmonic mean and arith metic mean in some conditions.

If a new process with burst time equal to $t_{n+1}$ arrives, with considering the extreme value of this parameter, we have two situations:

1. In equation 3 , we use extreme value for $t_{n+1}$ when it is very smaller than the others $\left(t_{n+1} \rightarrow 0\right)$. Based on this equation, if $t_{n+1}$ is very smaller than the average of previous values then the harmonic mean tends toward the $t_{n+1}$, but the arithmetic mean remains approximately near the previous value.

$$
\begin{aligned}
& \text { Harmonic Mean }= \\
& \qquad \lim _{t_{n+1} \rightarrow 0} \frac{n+1}{\frac{1}{t_{1}}+\frac{1}{t_{2}}+\ldots+\frac{1}{t_{n}}+\frac{1}{t_{n+1}}}=0
\end{aligned}
$$

2. In equation 4 , we use extreme value for $t_{n+1}$ when it is very larger than the others $\left(t_{n+1} \rightarrow \infty\right)$. Based on this equation, if $t_{n+1}$ is very larger than the average of previous values then the arithmetic mean tends toward the $\mathrm{t}_{\mathrm{n}+1}$, but the harmonic mean remains approximately near the previous value.

$$
\begin{aligned}
& \text { Harmonic Mean }= \\
& \qquad \lim _{t_{n+1} \rightarrow \infty} \frac{n+1}{\frac{1}{t_{1}}+\frac{1}{t_{2}}+\ldots+\frac{1}{t_{n}}+\frac{1}{t_{n+1}}}=\frac{n+1}{\frac{1}{t_{1}}+\frac{1}{t_{2}}+\ldots+\frac{1}{t_{n}}}
\end{aligned}
$$

This is approximately equal to the amount calculated in Equation 1. This means that if quantum time of new arrived process is too large then new harmonic mean is approximately equal to previous harmonic mean.

For example, we consider four processes P1, P2, P3 and $\mathrm{P} 4$ arriving at time 0 with burst time 20, 40, 50 and 80 respectively. The arithmetic mean of theme is 47.5 and harmonic mean of theme is 37.2. Now, suppose that new process P5 with burst time equal 2(Much smaller than the others) arrives, then the new arithmetic mean of five processes is 38.4 and new harmonic mean is 8.2.

Now suppose that new process P5 with burst time equal 150 (Much larger than the others) arrives, then the new arithmetic mean of five processes is 68 and new harmonic mean is 43.8 .

The above example shows that the harmonic mean is strongly influenced by too small a mounts and arithmetic mean influenced by too large amounts.

So if the burst time of new processes is very smaller than previous processes, it is better to use harmonic mean instead of arithmetic mean, because new means are closer to new arrived amounts and thus the average waiting time may be reduced, but if the arith metic mean is used, the new average will be close to previous amounts, and thus may increase the average waiting time. Similarly, if burst time of new processes is very larger than previous processes, it is better to use arithmetic mean instead of harmonic mean.

In this paper we will show that if the burst times of processes is heterogeneous, using the harmonic mean has high impact in reducing both average Waiting Time and average Turnaround Time.

\subsection{Pseudo Code of HARM Round Robin Algorithm}

In our algorithm, the arrival time of all processes is assumed to be zero. The inputs of algorith $m$ are burst time and number of processes ( $n) . \mathrm{q}_{\mathrm{t}}$ is the quantum time.

1. All the present processes are as signed to ready queue.

2. While(ready queue is not empty)

3. Calculate quantum time :

If (processes are heterogeneous and some of them were much smaller than the others) then

$$
\mathrm{q}_{\mathrm{t}}=\text { Harmonic Mean of burst times }
$$

else

If (processes are heterogeneous and some of them were much larger than the others) then

$$
\mathrm{q}_{\mathrm{t}}=\text { Arithmetic Mean of Burst times }
$$

4. Assign $\mathrm{q}_{\mathrm{t}}$ to processes

$$
\begin{gathered}
\mathrm{P}_{\mathrm{i}} \leftarrow \mathrm{q}_{\mathrm{t}} \\
\mathrm{i}=\mathrm{i}+1
\end{gathered}
$$

5. If(i<number of processes $)$ then goto step 4

6. If a new process is arrived:

Update ready queue and goto step 3

7. Calculate Average Waiting Time, Average Turnaround Time

8. End 


\section{Experimental Results}

In this section we just compare our algorithm with fixed quantum time Round Robin algorithm. To compare the proposed method with this method we study some cases. In the first case, consider five processes in table 1 . The burst times of these processes is given. The arrival time of these processes is considered zero. Burst times have been considered so that the burst time of fifth process had large difference with the previous processes (Much smaller than the others). In table 2 there are five processes that two of them are much smaller than the others. In table 3, seven heterogeneous processes are considered that three of them in an irregular sequence are much smaller than the others.

By analyzing data from these three tables we want to improve that if burst times of processes are heterogeneous and some of them are much smaller than the others it is better to use harmonic mean instead of arithmetic mean.

In this paper Waiting Time is defined as sum of the times that each process has to wait before it gets it's time slice and Turnaround time is defined as the time required for a particular process to complete, from submission time to completion. It should be noted that calculation steps of quantum time $\left(\mathrm{q}_{\mathrm{t}}\right)$ in Figure 1, 2, 3 is not shown and only calculated values of $\mathrm{q}_{\mathrm{t}}$ are displayed.

Table 1: Processes with burst time, last burst time is much smaller

\begin{tabular}{|c|c|c|}
\hline Process & Arrival Time & Burst Time \\
\hline P1 & 0 & 40 \\
\hline P2 & 0 & 60 \\
\hline P3 & 0 & 20 \\
\hline P4 & 0 & 55 \\
\hline P5 & 0 & 5 \\
\hline
\end{tabular}

Table 2: Five processes with burst time in random order

\begin{tabular}{|c|c|c|}
\hline Process & Arrival Time & Burst Time \\
\hline P1 & 0 & 70 \\
\hline P2 & 0 & 2 \\
\hline P3 & 0 & 55 \\
\hline P4 & 0 & 8 \\
\hline P5 & 0 & 60 \\
\hline
\end{tabular}

Table 3: Seven processes with burst time in random order

\begin{tabular}{|c|c|c|}
\hline Process & Arrival Time & Burst Time \\
\hline P1 & 0 & 1 \\
\hline P2 & 0 & 80 \\
\hline P3 & 0 & 7 \\
\hline P4 & 0 & 65 \\
\hline P5 & 0 & 70 \\
\hline P6 & 0 & 5 \\
\hline P7 & 0 & 60 \\
\hline
\end{tabular}

Table 4: Processes with burst time, last burst time is much larger

\begin{tabular}{|c|c|c|}
\hline Process & Arrival Time & Burst Time \\
\hline P1 & 0 & 40 \\
\hline P2 & 0 & 60 \\
\hline P3 & 0 & 20 \\
\hline P4 & 0 & 55 \\
\hline P5 & 0 & 200 \\
\hline
\end{tabular}

\subsection{Results of Table 1}

In this section we plot the Gantt diagram of processes with two scheduling method. Figure 1 shows the Gantt diagram of processes in table 1 based on arithmetic mean and the results of this diagram is shown in table 5 . Figure 2 shows the Gantt diagram of processes in table 1 based on harmonic mean and the results of this diagram is shown in table 6. In these tables, "TaT" is abbreviation for "Turnaround Time" and "WT" is abbreviation for "Waiting Time".

Here, we show steps of calculating quantum time for Figure 1 by using arithmetic mean. The numbers have been rounded.

$$
\begin{aligned}
& \mathrm{q}_{\mathrm{t}} \text { in step } 1=\frac{40+60+20+55+5}{5}=36 \\
& \mathrm{q}_{\mathrm{t}} \text { in step } 2=\frac{4+24+0+19+0}{3}=15.66 \approx 16 \\
& \mathrm{q}_{\mathrm{t}} \text { in step } 3=\frac{0+8+0+0+3}{2}=5.5 \approx 6
\end{aligned}
$$$$
\mathrm{q}_{\mathrm{t}} \text { in step } 4=\frac{0+2+0+0+0}{1}=2
$$

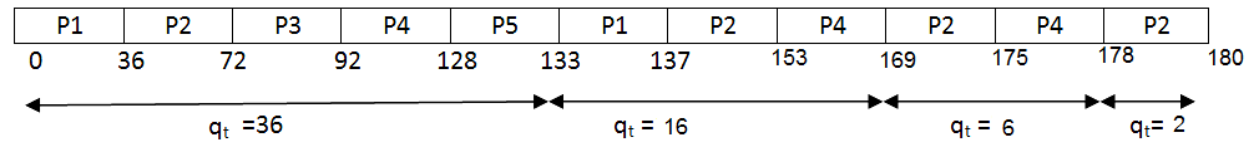

Fig. 1: Gantt diagram of processes in table 1 based on arithmetic mean

Table 5: Results obtained from Figure 1

\begin{tabular}{|c|c|}
\hline Algorithm & Dynamic Round Robin based on arithmetic mean \\
\hline $\mathrm{CS}$ & 10 \\
\hline $\mathrm{AVR}(\mathrm{TaT})$ & 144 \\
\hline $\mathrm{AVR}(\mathrm{WT})$ & 108 \\
\hline
\end{tabular}


And here, steps of calculating quantum time for Figure 2 based on harmonic mean are shown. The numbers have been rounded.

$$
\mathrm{q}_{\mathrm{t}} \text { in step } 1=\frac{5}{\frac{1}{40}+\frac{1}{60}+\frac{1}{20}+\frac{1}{55}+\frac{1}{5}}=16.13 \approx 16
$$$$
\mathrm{q}_{\mathrm{t}} \text { in step } 2=\frac{4}{\frac{1}{24}+\frac{1}{44}+\frac{1}{4}+\frac{1}{39}+0}=11.76 \approx 12
$$

$$
\mathrm{q}_{\mathrm{t}} \text { in step } 3=\frac{3}{\frac{1}{12}+\frac{1}{32}+0+\frac{1}{27}+0}=19.78 \approx 20
$$$$
\mathrm{q}_{\mathrm{t}} \text { in step } 4=\frac{2}{0+\frac{1}{12}+0+\frac{1}{7}+0}=8.84 \approx 9
$$

\begin{tabular}{|c|c|c|c|c|c|c|c|c|c|c|c|c|c|c|}
\hline P1 & P2 & P3 & P4 & P5 & $\mathrm{P} 1$ & P2 & P3 & P4 & P1 & P2 & P4 & $\mathrm{P} 2$ & P4 & P2 \\
\hline 0 & 16 & 32 & 48 & 64 & 69 & 81 & 93 & $\frac{1}{97}$ & 109 & $\frac{1}{121}$ & $\frac{1}{141}$ & 161 & 170 & $\frac{1}{177}$ \\
\hline
\end{tabular}

$\mathrm{q}_{\mathrm{t}}$ in step $5=\frac{1}{0+\frac{1}{3}+0+0+0}=3$

Fig 2: Gantt diagram of processes in table 1 based on harmonic mean

Table 6: Results obtained from Figure 2

\begin{tabular}{|c|c|}
\hline Algorithm & $\begin{array}{c}\text { Dynamic Round Robin based on } \\
\text { harmonic mean }\end{array}$ \\
\hline CS & 14 \\
\hline AVR(TaT) & 128.8 \\
\hline AVR(WT) & 72.8 \\
\hline
\end{tabular}

\subsection{Results of Table 2}

Results for these five processes using three methods have been compared to each other in table 7 . Consider that the burst times of these processes are heterogeneous. In the round robin scheduling method fixed quantum time is assumed equal to 20 .

Table 7: Compared results obtained from table 2

\begin{tabular}{|c|c|c|c|}
\hline $\begin{array}{c}\text { Round Robin } \\
\text { Algorithm }\end{array}$ & Fixed quantum time with qt=20 & $\begin{array}{c}\text { Dynamic quantum time based } \\
\text { on arithmetic mean }\end{array}$ & $\begin{array}{c}\text { Dynamic quantum time based } \\
\text { on harmonic mean }\end{array}$ \\
\hline AVR(TaT) & 103 & 102.8 & 84.2 \\
\hline AVR(WT) & 64.4 & 63.8 & 45.2 \\
\hline
\end{tabular}

Table 8: Compared results obtained from table 3

\begin{tabular}{|c|c|c|c|}
\hline $\begin{array}{c}\text { Round Robin } \\
\text { Algorithm }\end{array}$ & Fixed quantum time with $\mathbf{q}=\mathbf{3 0}$ & $\begin{array}{c}\text { Dynamic quantum time based } \\
\text { on arithmetic mean }\end{array}$ & $\begin{array}{c}\text { Dynamic quantum time based } \\
\text { on harmonic mean }\end{array}$ \\
\hline $\mathrm{AVR}(\mathrm{TaT})$ & 176.28 & 150.57 & 126.42 \\
\hline $\mathrm{AVR}(\mathrm{WT})$ & 126.57 & 103.72 & 82.42 \\
\hline
\end{tabular}

\subsection{Results of Table 3}

Results for these seven processes using three methods have been compared to each other in table 8. Consider that the burst times of these processes are heterogeneous. In the round robin scheduling method, fixed quantum time is as sumed equal to 30 .

\subsection{Results of Table 4}

In table 4 we have the same processes in table 1 except that the process P5 has burst time equal to 200(Much larger than the others). The quantum time for Round Robin algorithm with fixed quantum time is assumed to 20. Figure 3 shows the Gantt diagram of processes in table 4 based on arithmetic mean and the results of this diagram is shown in table 9.

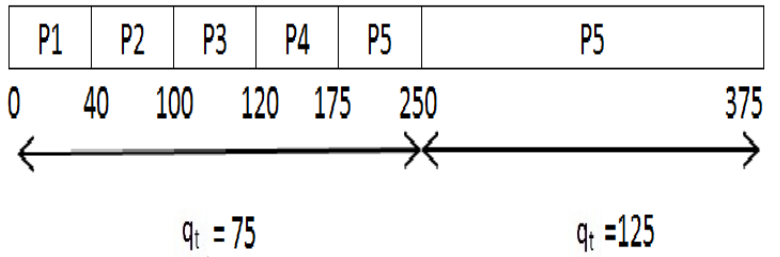

Fig. 3: Gantt diagram of processes in table 4 based on arithmetic ean 
Table 9: Compared results obtained from table 4

\begin{tabular}{|c|c|c|c|}
\hline Round Robin Algorithm & Fixed quantum time with $\mathbf{q}_{\mathbf{t}}=\mathbf{2 0}$ & $\begin{array}{c}\text { Dynamic quantum time based } \\
\text { on arithmetic mean }\end{array}$ & $\begin{array}{c}\text { Dynamic quantum time } \\
\text { based on harmonic mean }\end{array}$ \\
\hline AVR(TaT) & 194 & 136.8 & 195.8 \\
\hline AVR(WT) & 119 & 87 & 143.8 \\
\hline
\end{tabular}

As can be seen for these processes arithmetic mean provides better results than harmonic mean.

\section{Conclusion}

In this paper we proposed a new algorithm to compute quantum time in round robin algorithm. This approach is based on harmonic-arithmetic mean that we called it HARM. According to this algorithm when burst time of processes is heterogeneous and burst time of new processes is very smaller than previous it is better to use harmonic mean to calculating quantum time and if burst time of new processes is very larger than previous it is better to use arithmetic mean to calculating quantum time. A comparative study of proposed algorithm and Round Robin algorithm with fixed quantum time, Dynamic quantum time based on arithmetic mean and dynamic quantum time based on harmonic mean is made. It is concluded from these tables that if burst time of new processes is very smaller than previous entries it is better to use harmonic mean to calculating quantum time and if it is very larger than previous entries it is better to use harmonic mean to calculating quantum time. According to the results obtained from tables we conclude that by applying proposed dynamic quantum time in this paper, average waiting time and average turnaround time dramatically decrease because with decreasing of burst times of new arrived processes the quantum time in harmonic method gets smaller and this lead to decreasing of average waiting time of processes. In very situations the burst time of processes is not heterogeneous and in these situations it is better to use dynamic Round Robin scheduling algorithm that proposed in this paper. For the future perspective we would like to apply arrival time with burst time to analyze our algorithm. Another future work is to exert the proposed algorithm for real time systems and you can analysis this research for improvement.

\section{References}

[1] Rami J. Matarneh. Self-Adjustment Time Quantum in Round Robin Algorithm Depending on Burst Time of Now Running Processes, American J. of Applied Sciences 6(10): 1831-1837, 2009.

[2] H. S. Behera, Rakesh Mohanty, Debashree Nayak. A New Proposed Dynamic Quantum with ReAdjusted Round Robin Scheduling Algorithm and Its Performance Analysis, International Journal of
Computer Applications, Vol. 5, No. 5, August 2010.

[3] Abbas Noon Ali Kalakech Seifedine Kadry. A New Round Robin Based Scheduling Algorithm for Operating Systems-Dynamic Quantum Using the Mean Average, IJCSI International Journal of Computer Science Issues, Vol. 8, Issue 3, No. 1, May 2011.

[4] Helmy, T. and A. Dekdouk, 2007. Burst Round Robin as a Proportional-share Scheduling Algorithm, IEEEGCC,http://eprints.kfupm.edu.sa/1462.

[5] Prof. Rakesh Mohanty, Prof. H. S. Behera Khusbu Patwari, Manas Ranjan Das, Monisha Dash, Sudhashree. Design and Performance Evaluation of a New Proposed Shortest Remaining Burst Round Robin (SRBRR) Scheduling Algorithm, Department of Computer Science and Engineering Veer Surendra Sai University of Technology, Burla, Sambalpur, Orissa, India, 2010.

[6] Prof. Rakesh Mohanty, Manas Ranjan Das, M.Lakshimi prasanna, Sudhashree Students. Design and Performance Evaluation of a New Proposed Fittest Job First Dynamic Round Robin (FJFDRR) Scheduling Algorithm, International Journal of Computer Information Systems, vol. 2, No. 2, 2011.

[7] Saroj Hiranwal. Adaptive round robin scheduling using shortest burst approach based on smart time slice, International Journal of Computer Science and Communication, vol2, No.2, July-December 011, pp.319-323.

[8] Mark W. Shirley. Review of Statistical Analysis, Appendix VI, Cost of Capital: Applications and Examples, Third Edition by Shannon Pratt and Roger J. Grabowski, copyright 2008 by John Wiley and Sons, p. 738.

[9] Shannon Pratt. The Market Approach to Valuing Businesses, Second Edition, copyright 2005 by John Wiley \& Sons, p. 140.

[10] Pallab banerjee, probal banerjee, shweta sonali dhal. Comparative Performance Analysis of Average Max Round Robin Scheduling Algorithm (AMRR) using Dynamic Time Quantum with Round Robin Scheduling Algorithm using static Time Quantum, IJITEE,ISSN: 2278-3075, Volume-1, Is sue-3, August 2012.

[11] Operating Systems, 3rd Ed., H.M.Deitel, P.J.Deitel, 
D.R.Choffnes .ISBN 978-81-317-1289-4.

[12] Operating System Concepts, 8th Ed., Abraham Silberschatz, Peter B. Galvin, Grege Gagne. ISBN 978-81-265-2051-0.

[13] Tanebaun, A.S. 2008. Modern Operating Systems. 3rd Edn., Prentice Hall, ISBN: 13:9780136006633, pp: 1104.

[14] C. L. Liu and James W. Layland. Scheduling Algorithms for Multiprogramming in a Hard-RealTime Environment, Journal of the ACM (JACM), Vol. 20, Issue 1, January, 1973.

[15] Sharma R (2008). Some more inequalities for arithmetic mean harmonic mean and variance, $\mathrm{J}$ Math Inequal 2 (1) 109-114.

[16] B. C. Carlson. Algorithms involving arith metic and geometric means, A mer. Math. Monthly 78 (1971), 496-505. MR 0283246.

[17] Christopher D. Manning, Prabhakar Raghavan and Hinrich Schütze. Introduction to Information Retrieval, Cambridge University Press, 2008.

\section{Authors' Profiles}

Ashkan Emami Ale Agha: Graduate Student for computer engineering in Islamic Azad University, Science and Research Branch, Tehran, Iran, interested in computer architecture, Operating Systems design and Sensor Networks.

Somayyeh Jafarali Jassbi: Assistant professor of Department of Computer Engineering in Islamic Azad University, Science and Research Branch, Tehran, Iran, interested in computer architecture, Residue Number Systems, VLSI design.

How to cite this paper: Ashkan Emami Ale Agha, Somay yeh Jafarali Jassbi,"A New Method to Improve Round Robin Scheduling Algorithm with Quantum Time Based on Harmonic-Arithmetic Mean (HARM)", International Journal of Information Technology and Computer Science(IJITCS), vol.5, no.7, pp.56-62, 2013. DOI: 10.5815/ijitcs.2013.07.07 